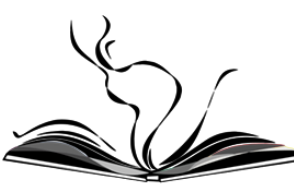

Vivian Urquidi

Maria Cristina Cacciamali ${ }^{2}$

Bruno Massola Moda ${ }^{3}$

Universidade de São Paulo, Brasil

Versión en español

English version

\title{
Um lugar para memórias (Carta às leitoras e aos leitores),
}

Ao finalizar 2020, um ano de dor e perplexidade, a Brazilian Journal of Latin American Studies gostaria de presentear suas leitoras e seus leitores com uma nova edição da sua revista científica orientada para a publicação de estudos sobre a América Latina e o Caribe em diversas perspectivas que traduzem a riqueza da região, assim como as realidades complexas dos países do nosso continente.

Pensar a América Latina e o Caribe requer interpretar a situação de dependência regional, num sistema mundo em que os poderes econômicos e políticos já definiram suas posições e seus movimentos no tabuleiro da geopolítica internacional. Exige conceber também a integração nacional em países marcados por históricas feridas coloniais, e como tal garantem a estrutura das hierarquias sociais em que as classes se

\footnotetext{
1 Doutora em Sociologia pela Universidade de São Paulo e Pós-doutora no Centro de Estudos Sociais da Universidade de Coimbra. É Professora adjunta da Universidade de São Paulo no Curso de Gestão de Políticas Públicas e nos Programas de Pós-graduação Integração da América Latina e de Estudos Culturais. E-mail: vurquidi@usp.br

2 Doutora em Economia pela Universidade de São Paulo e Pós-doutora no Instituto de Tecnologia de Massachusetts e na Universidad de Nueva México. É Professora titular da Universidade de São Paulo na Faculdade de Economia e Administração e no Programas de Pós-graduação Integração da América Latina E-mail: cciamali@uol.com.br

${ }^{3}$ Doutorando pelo Programas de Pós-graduação Integração da América Latina da Universidade de São Paulo. E-mail: bruno.moda@hotmail.com
} 
relacionam com critérios de raça, gênero, sentimentos nacionalistas ou regionalistas, quando não inclusive religiosos.

Estas são as questões que trata a edição 38 da BJLAS e são também o motivo que nos induz a abrir o número com o artigo O CINEMA DE SARA GÓMEZ: UMA LEITURA SOBRE O CONTEXTO PÓS-REVOLUÇÃO EM CUBA.

A crítica inspiradora do filme De Cierta Manera da cineasta negra cubana Sara Gómez resulta do trabalho de pesquisa da doutora em História Social, Cleonice Elias Silva, que com sensibilidade estética e política apresenta o cenário pós-revolucionário de Cuba, e das revoluções que a Revolução não conseguiu realizar. A partir de um filme da década de 1970, a autora interpreta problemáticas que estão na ordem do dia em países do resto do continente: racismo estrutural, violência de gênero, tensões entre o tradicional e o moderno, além de outros debates sobre as identidades que ultrapassam a situação de classe e que subjazem nas grandes questões pendentes da América Latina, negra, indígena e diversa.

A análise dialoga com as epistemologias negras e os estudos culturais, e nesta seara se afina com as duas discussões essencialmente teóricas que apresentamos a seguir neste número da BJLAS. Ambos enfoques, como se verá, têm críticas que a teoria social conceitua como decoloniais. A primeira se desenvolve no campo da comunicação e da cultura. A segunda tem um enfoque a partir das arenas da história e da filosofia.

Vejamos o primeiro estudo, AMÉRICA LATINA POR UMA EPISTEMOLOGIA DECOLONIAL DA COMUNICAÇÃO, de Bruno Santos N. Dias, Doutorando em Ciências da Comunicação. A partir do campo da Comunicação e da Cultura, o autor debate as teorias clássicas sobre a produção mediática e a indústria cultural, cujos pressupostos instrumentalizam todos os conhecimentos das regiões periféricas do 
sistema e as criações culturais produzidas pelos povos subalternizados. 0 autor leva o conceito de colonialidade do saber - preconizado pelo projeto Modernidade/Colonialidade - ao centro do debate sobre as teorias da comunicação na América Latina e no Caribe, oferecendo como alternativa uma epistemologia dialógica, orgânica e plural, ancorada nos saberes diversos, distintos e 'encantados' que se produzem nas periferias dos países da região, como movimentos intelectuais de resistência e desobediência teórica, política, cultural e prática.

Numa trajetória semelhante, Adriana de Carvalho Alves Braga e Christian Fernando dos Santos Moura, doutores em Pedagogia e em Artes, expõem o projeto eurocêntrico da história oficial, que invisibiliza e silencia as vozes das vítimas do colonialismo e dos poderes coloniais vigentes na América Latina. O desafio do artigo é fazer dialogar as críticas decoloniais com as notas de Walter Benjamin no Conceito de História, notas que denunciam a primazia da história dos vencedores. Mas o artigo vai além: articula ambas perspectivas com a crítica inaugurada pelo marxismo revolucionário de José Carlos Mariátegui, que no início do século XX já vislumbrava o potencial protagonista dos povos indígenas como atores da sua transformação política e social. Deste exercício intelectual resulta o título eloquente AS VOZES EMUDECIDAS: UM DEBATE SOBRE AS FONTES CONTRA-HEGEMÔNICAS PARA A COMPREENSÃO DA AMÉRICA LATINA. O desafio intelectual se completa quando os autores analisam as crônicas de Guamán Poma de Ayala - o indígena nascido no século XVI que descreve e desenha a violência colonial - como fonte de dados históricos claramente descolonizadora e anticolonial, e como narrativa legítima e contestadora da história oficial.

O resgate e o desafio de preservação da memória são parte do sucesso das lutas políticas contra as formas históricas de opressão. 0 direito à memória é componente central da busca por justiça dos povos que passaram por grandes traumas depois de episódios de violências, 
principalmente por agentes do estado, e que com resistência buscam a transição para uma democracia duradoura.

É nesse pano de fundo, que os trabalhos a seguir devem ser lidos, como projetos intelectuais e políticos de resgate da memória de um dos episódios mais abjetos das histórias contemporâneas latino-americanas: a promoção de ditaduras civil-militares que se deu articuladamente nos países da região, e que durou por mais de duas décadas na segunda metade do século XX.

Não é de estranhar, pois, que Walter Benjamin tenha sido também inspirador dos artigos que apresentamos como trabalhos de resgate da memória pois, "é papel do historiador retomar o passado dos que foram subjugados ou vencidos, contrapondo-se à história apresentada como progresso."

Encontramos essa citação no artigo LUGARES DE MEMÓRIA DAS DITADURAS CIVIS-MILITARES LATINO-AMERICANAS: ARQUITETURA E INSCRIÇÕES DE SENTIDO. O artigo apresenta os resultados de uma pesquisa original que analisa o legado das ditaduras a partir de três instalações arquitetônicas criadas no Chile, na Argentina e no Brasil, com a finalidade de guardar registros e manter acesas as lembranças dos regimes que se valeram de tortura, assassinatos, sequestros e desaparecimentos como modo de controle e exercício do poder estatal. As instalações formam parte das lutas por consolidar as frágeis democracias na região e nasceram de demandas populares e da ação consciente dos governos progressistas destes três países. Os autores que ainda nos brindam com ilustrações fotográficas e quadros comparativos dessas iniciativas são os mestrandos em Ciências da Informação, Caio Vargas Jatene, e em Arquitetura e Urbanismo, Luiz de Lucca Neto.

Também é um resgate da memória contra as ditaduras latino-americanas o artigo escrito por Ana Carolina Contin Kosiak, mestre 
em Direito, e Marcos Gonçalves, doutor em História. O artigo resulta da análise dos "arquivos da repressão", registros dos órgãos de segurança brasileiros nas fronteiras que contêm informações sobre os argentinos que fugiam ao Brasil por causa do recrudescimento da ditadura na Argentina de final de década de 1970. O artigo COOPERAÇÃO E TRÂNSITO ENTRE AS DITADURAS ARGENTINA E BRASILEIRA: O REGISTRO DE PESSOAS REFUGIADAS ARGENTINAS EXILADAS NO BRASIL (1977 - 1979) merece destaque pela ampla pesquisa documental em registros que foram elaborados pelos agentes de controle das fronteiras durante um período crítico da ditadura argentina. Inclui dados estatísticos colhidos sobre a composição dos perseguidos e refugiados, o que incluía mulheres, crianças e famílias inteiras. O trabalho é também digno de menção porque trilha as estratégias de colaboração menos conhecidas entre as ditaduras latino-americanas para perseguir internacionalmente seus opositores políticos. No caso do Brasil, a colaboração dos militares brasileiros com a ditadura da Argentina ocorria inclusive quando o regime brasileiro experimentava certa abertura política e aparentava disposição humanitária junto aos organismos internacionais de proteção aos refugiados políticos da ONU.

Das relações internacionais entre aparatos de repressão, saímos para adentrar nos projetos econômicos e de integração regional como política externa de Estado. Este é o tema do artigo IIRSA, COSIPLAN E A ATUAÇÃO BRASILEIRA NA CONSOLIDAÇÃO DA INTEGRAÇÃO DE INFRAESTRUTURA NA AMÉRICA DO SUL. A análise das iniciativas de integração a partir de projetos de engenharia civil e infraestrutura servem de sustento concreto para testar a hipótese do subimperialismo brasileiro na América do Sul, concluindo que, apesar dos benefícios para a consolidação internacional das empresas brasileiras a partir dos acordos estabelecidos com os países vizinhos no âmbito da infraestrutura e construção civil, não é possível considerar que o propósito do Brasil tenha sido consolidar um subimperialismo econômico. Para corroborar esta 
conclusão, os autores trazem tabelas estatísticas e gráficos que atestam que os dados de investimentos do Brasil são "inexpressivos" quando comparados aos dos países asiáticos ou da União Europeia. $\bigcirc$ artigo é escrito em coautoria por Ana Karolina Morais Silva, Jéssica Maria Grassi e Lucas Kerr Oliveira, pesquisadores especialistas em Relações Internacionais na América Latina. Concluem os autores que a explicação mais justa para a atuação brasileira nos países vizinhos seria considerar sua política externa como um projeto de liderança regional pró-integração que foi desenvolvido ao longo de duas décadas.

O padrão de reprodução capitalista e a leitura crítica sobre a dependência econômica regional são temáticas do artigo desenvolvido pelo doutor em economia, José Alex Rego Soares, sob o título DEPENDÊNCIA FINANCEIRA LATINO-AMERICANA NO SÉCULO XIX: 0 PAPEL DA CITY NA CONDUÇÃO DA INSERÇÃO REGIONAL NO O SISTEMA FINANCEIRO INTERNACIONAL. A dependência regional é analisada a partir do século XIX, quando da formação das nascentes repúblicas, e da inserção progressiva das novas economias no capitalismo a se tornar globalizado. Isto ocorreu mediante empréstimos e incentivos promovidos pelo sistema financeiro britânico para os regimes republicanos da América Latina. A lógica deste engajamento com o mercado financeiro teria ocorrido sob as circunstâncias herdadas do período colonial. Assim, o artigo demonstra como os vínculos econômicos com as metrópoles foram mudados pelo regime do sistema financeiro britânico e pela relação desta instância com os grupos econômicos locais. Conclui-se que, se por um lado as nascentes repúblicas necessitavam de recursos econômicos externos, por outro lado os capitais financeiros contribuíram para criar dívidas que, em última instância, favoreceram o estrangulamento das condições de desenvolvimento futuro e de integração regional.

Os próximos artigos apresentados pela BJLAS colocam em diálogo o campo da geografia com os estudos sobre a ecologia e economia políticas, 
e com os debates sobre desenvolvimento, sociedade e cultura. Isso porque são artigos que discorrem teoricamente e mediante estudos de caso a problemática da agricultura na América Latina, uma temática tão antiga e atual quanto as tensões pelo território e pelas autonomias.

Nas últimas décadas, observou-se um incremento dos estudos sobre a agricultura relacionados com as crises socioambientais e culturais. E à medida em que o debate sobre o habitat e a qualidade de vida tensiona a lógica do desenvolvimento, a gestão pública se viu forçada a considerar a agricultura dentro do âmbito urbano como questão central das políticas de urbanização.

Colocou-se assim um desafio não apenas político, como também teórico sobre as novas questões que a urbanização e o meio ambiente têm proposto para as tarefas já complexas da administração pública, obrigando gestores e intelectuais a dialogar com novos campos de conhecimento.

É do desafio de pensar a agricultura urbana a partir de novos campos da ciência social e com novos instrumentos conceituais que Henrique Freitas Alves, mestrando em geografia humana, escreve o artigo ECOLOGIA POLÍTICA E AGRICULTURA URBANA NA AMÉRICA LATINA: REFLEXÕES TEÓRICAS POR UMA APROXIMAÇÃO. O argumento que se desenvolve neste trabalho é que a agricultura urbana vem exigindo aos estudos e às políticas de urbanização uma abertura para incorporar questões fundamentais suscitadas pela ecologia política urbana. Com uma sistematização teórica importante, o artigo traz um panorama dos estudos sobre a agricultura urbana como "forma de reapropriação social da natureza", e é neste nível que a ecologia política se transforma em ferramenta central para dar respostas às novas questões da desigual distribuição do espaço urbano e do direito à cidade. São tópicos novos que nos remetem a pensar filosófica e teoricamente numa nova categoria de justiça: a "justiça espacial". 
Em uma perspectiva distinta de análise da agricultura na América Latina, os dois estudos que se seguem nos colocam perante o avanço da agroindústria e o agronegócio, em áreas camponesas e em novas terras transformadas para a produção intensiva e extensiva para o mercado globalizado. Os artigos se complementam como estudos de caso sobre a Colômbia, país paradigmático para entender as novas ruralidades.

Começaremos com os estudos da doutoranda Rosa Inés Babilonia Ballesteros e o doutor Júlio César Suzuki, ambos da área da Geografia Humana, que discutem aquilo que o pensamento geográfico denomina como as novas ruralidades, isto é, os efeitos das atividades econômicas que transformam os espaços rurais outrora integrados pela atividade amparada na tradição agrícola dos camponeses. O cenário desta análise é a Colômbia, país em que nas últimas décadas houve mudanças sociais e culturais profundas ocasionadas pela transição da agricultura camponesa à agricultura comercial e de exportação. Assim, o cenário histórico é o da expansão das políticas de neoliberalização ao espaço rural, com a consequente desagregação social e cultural que essa nova lógica impõe aos camponeses. Cabe destacar, que o artigo resulta de uma pesquisa de fôlego com entrevistas e imersão no campo para colher dados qualitativos de grande sensibilidade metodológica que permite entender as consequências da nova ruralidade sobre os territórios e a vida das comunidades camponesas. Com este enfoque, o artigo se intitula $\boldsymbol{A}$ ABORDAGEM QUALITATIVA E SUAS CONTRIBUIÇÕES PARA ESTUDAR O ESPAÇO RURAL: UMA EXPERIÊNCIA A PARTIR DA NOVA RURALIDADE NA COLÔMBIA.

O segundo estudo complementa o anterior com um artigo intitulado GLOBALIZAÇÃO E RESILIÊNCIA NA SAVANA SAZONAL DE PUERTO GAITÁN, META, COLÔMBIA: REFLEXÕES TEÓRICAS E METODOLÓGICAS. Partindo de preocupações epistêmicas semelhantes -o avanço do agronegócio sobre novas fronteiras agrícolas- este estudo inova ao 
destacar, com dados históricos, o papel central do governo e das instituições internacionais que promovem o desenvolvimento regional no sucesso da nova ruralidade. O segundo aspecto particular deste trabalho é que destaca o avanço dos capitais do agronegócio sobre terras inabitadas ou consideradas não apropriadas para a agricultura camponesa, justificando deste feito a ocupação pela indústria agropecuária baseada na biotecnologia. Ocorre porém, como é demonstrado pelo autor, Carlos Enrique Castro Méndez, doutor em Geografia, que muito embora estas terras não fossem tradicionalmente ocupadas pela agricultura camponesa, elas têm um lugar na preservação do ecossistema local e da relação dos seres que aí habitam com seu entorno natural. Da exploração destas terras se desprendem formas de segregação espacial e níveis concretos de perda de governança territorial, entre outras consequências da desterritorialização produzida pelos fenômenos econômicos globais que incidem, inclusive provocando riscos ambientais futuros imponderáveis.

O último artigo desta edição da BJLAS tem também a Colômbia como o locus do tema abordado, especificamente a cidade de Medellín e as políticas de segurança cidadã implementadas com resultados concretos na diminuição da violência urbana. A tese que se defende é que as competências e autonomias que se outorgaram à gestão municipal desde a década de 1990 permitiram as significativas cifras que reduziram, por exemplo, 90\% dos homicídios no município. Desse modo, há um caráter pedagógico fundamental na análise da experiência de Medellín e que este artigo expressa com precisão no título: O PAPEL DO MUNICíPIO NA PROMOÇÃO DA SEGURANÇA CIDADÃ: POSSÍVEIS LIÇÕES DE MEDELLÍN. O aspecto mais relevante desta abordagem no campo das Ciências Jurídicas e do Direito é que a autora, pesquisadora de nível de mestrado Luiza Veronese Lacava, demonstra que a construção de uma política de segurança teve como requisito a participação cidadã e amparo dos debates do Acordo de Paz, que foi consagrado finalmente em 2016, depois de anos de construção tensa com participação não apenas do governo e 
das FARC, mas de setores da população. Entre outros aspectos, o Acordo garante mecanismos de proteção da participação popular e das manifestações políticas públicas. Logo, a segurança deixa de ser questão de ordem pública e repressão para estar orientada pelos pressupostos de cidadania e participação.

Complementando como sempre os artigos da BJLAS, incluímos a resenha de um livro construído com relatos fictícios e reais sobre a Literatura Nazi na América Latina (Roberto Bolaño). O tema da resenha fecha de modo impecável um número destinado a lembrar as mazelas sempre atualizadas da violência estrutural que forma as mediações políticas entre o Estado e a Sociedade. Este produto da literatura latino-americana foi resenhado pelo doutor em História Lincoln Secco.

Español

\section{Un lugar para las memorias (Carta a lectores y lectores),}

Al cabo de 2020, un año de dolor y perplejidad, la Brazilian Journal of Latin American Studies quisiera brindar a sus lectoras y lectores esta nueva edición de su revista científica destinada a publicar estudios sobre América Latina y el Caribe cuyas diferentes perspectivas traducen la riqueza de la región, pero también las complejas realidades de los países de nuestro continente.

Pensar América Latina y el Caribe requiere interpretar la situación de dependencia regional, en un sistema mundial en el que los poderes económicos y políticos ya han definido sus posiciones y movimientos en el tablero de la geopolítica internacional. También requiere una concepción sobre la integración nacional en países históricamente marcados por las heridas coloniales, determinantes de las jerarquías sociales en las que las 
clases se relacionan bajo criterios de raza, género, sentimientos nacionalistas o regionalistas, si no religiosos.

Estas son las cuestiones que aborda la $38^{a}$ edición de BJLAS y son también el motivo que nos lleva a abrir el número con el artículo EL CINE DE SARA GÓMEZ: UNA LECTURA SOBRE EL CONTEXTO POSTERIOR A LA REVOLUCIÓN EN CUBA.

La inspiradora crítica de la película De Cierta Manera de la cineasta negra cubana Sara Gómez resulta de la labor investigadora de la doctora en Historia Social, Cleonice Elias Silva, quien con sensibilidad estética y política presenta el escenario posrevolucionario de Cuba, y las revoluciones que la Revolución no pudo lograr. A partir de una película de la década de 1970, la autora interpreta temas que están a la orden del día en países del resto del continente: racismo estructural, violencia de género, tensiones entre lo tradicional y lo moderno, además de otros debates sobre las identidades que van más allá de la situación de clase y que subyacen a los grandes temas pendientes de una América Latina negra, indígena y diversa.

El análisis dialoga con las epistemologías negras y los estudios culturales, y en este ámbito está en sintonía con las dos discusiones esencialmente teóricas que presentamos a continuación en este número de la revista. Ambos enfoques, como se verá, tienen críticas que la teoría social conceptualiza como decoloniales. El primero se desarrolla en el campo de la comunicación y la cultura. El segundo se centra en las arenas de la historia y la filosofía.

Veamos el primer estudio, AMÉRICA LATINA POR UNA EPISTEMOLOGÍA DECOLONIAL DE LA COMUNICACIÓN del investigador de doctorado Bruno Santos N. Dias. Desde el campo de la Comunicación y la Cultura, el autor debate teorías clásicas sobre la producción mediática y la industria cultural, cuyos supuestos instrumentalizan todo el conocimiento de las regiones periféricas del sistema y las creaciones culturales producidas por 
los pueblos avasallados. El autor lleva el concepto de colonialidad del saber -pugnado por el proyecto Modernidad / Colonialidad- al centro del debate sobre las teorías de la comunicación en América Latina y el Caribe. Y ofrece como alternativa una epistemología dialógica, orgánica y plural, anclada en saberes diversos, diferenciados y "encantados". Saberes que se producen en las periferias de los países de esta región, como movimientos intelectuales de resistencia y desobediencia teórica, política, cultural y práctica.

En una trayectoria similar, Adriana de Carvalho Alves Braga y Christian Fernando dos Santos Moura, doctores en Pedagogía y Artes, exponen el proyecto eurocéntrico de la historia oficial, que invisibiliza y silencia las voces de las víctimas del colonialismo y de los poderes coloniales en América Latina. El desafío del artículo es conseguir que dialoguen las críticas decoloniales con las notas de Walter Benjamin en el Concepto de Historia, notas que denuncian la primacía de la historia de los vencedores. Pero el artículo va más allá: articula ambas perspectivas con la crítica inaugurada por el marxismo revolucionario José Carlos Mariátegui, quien ya a principios del siglo XX vislumbraba al potencial protagonista de los pueblos indígenas como actores de su transformación política y social. Este ejercicio intelectual da como resultado el elocuente título LAS VOCES MUDAS: UN DEBATE SOBRE LAS FUENTES CONTRAHEGEMÓNICAS PARA ENTENDER AMÉRICA LATINA. El desafío intelectual se completa cuando los autores analizan las crónicas de Guamán Poma de Ayala - el indígena nacido en el siglo XVI que describe y dibuja la violencia colonial como una fuente de datos históricos claramente descolonizadora y anticolonial, y como una narrativa legítima y desafiante de la historia oficial.

El rescate y el desafío de preservar la memoria son parte del éxito de las luchas políticas contra las formas históricas de opresión. El derecho a la memoria es un componente central de la búsqueda de justicia para las personas que han experimentado grandes traumas luego de episodios de 
violencia, especialmente por agentes del Estado, y que resistiendo, buscan la transición para una democracia duradera.

Es en este contexto que deben leerse los siguientes trabajos, como proyectos intelectuales y políticos para rescatar la memoria de uno de los episodios más abyectos de la historia latinoamericana contemporánea: la promoción de dictaduras cívico-militares que se desarrollaron articuladamente en los países de la región, y que duraron más de dos décadas en la segunda mitad del siglo XX.

No es de extrañar, por tanto, que Walter Benjamin también sirviera de inspiración para los artículos que presentamos como obras que rescatan la memoria porque, "es función del historiador retomar el pasado de quienes fueron subyugados o derrotados, oponiéndose a la historia presentada como progreso".

Encontramos esta cita en el artículo LUGARES DE MEMORIA DE LAS DICTADURAS CÍVICO-MILITARES EN LATINOAMÉRICA, ARQUITECTURA E INSCRIPCIONES DE SENTIDO. El artículo presenta los resultados de una investigación original que analiza el legado de las dictaduras a partir de tres instalaciones arquitectónicas creadas en Chile, Argentina y Brasil, cuyo propósito es dejar registros y mantener viva la memoria de los regímenes que utilizaron la tortura, los asesinatos, secuestros y desapariciones como forma de control y de ejercicio del poder estatal. Las instalaciones son parte de la lucha por la consolidación de las frágiles democracias de la región y nacieron de las demandas populares y de la acción consciente de los gobiernos progresistas de estos tres países. Los autores que aún nos brindan ilustraciones fotográficas y cuadros comparativos de estas iniciativas son los investigadores de Maestría en Ciencias de la Información, Caio Vargas Jatene, y en Arquitectura y Urbanismo, Luiz de Lucca Neto.

El artículo de Ana Carolina Contin Kosiak, master en Derecho, y Marcos Gonçalves, doctor en Historia, es también un rescate de la memoria frente a las dictaduras latinoamericanas. El artículo es el resultado del análisis de 
los "archivos de la represión", registros de las agencias de seguridad brasileñas en las fronteras que contienen informaciones sobre los argentinos que huyeron a Brasil debido a la agudización de la dictadura en Argentina a fines de la década de 1970. El artículo COOPERACIÓN $\boldsymbol{Y}$ TRÁNSITO ENTRE LAS DICTADURAS ARGENTINA Y BRASILEÑA: EL REGISTRO DE LOS REFUGIADOS ARGENTINOS EXILIADOS EN EL BRASIL (1977 - 1979) es merecedor de destaque por la amplia investigación documental en registros que fueron elaborados por agentes de control de fronteras durante un período crítico de la dictadura argentina. Incluye datos estadísticos recopilados sobre la composición de los perseguidos y refugiados, que incluyen mujeres, niños y familias enteras. El trabajo también es digno de mención porque rastrea las estrategias de colaboración menos conocidas entre las dictaduras latinoamericanas para perseguir a sus oponentes políticos a nivel internacional. En el caso de Brasil, la colaboración de los militares brasileños con la dictadura argentina se produjo incluso cuando el régimen brasileño experimentaba una cierta apertura política y se mostraba dispuesto a políticas humanitarias de acuerdo a los organismos internacionales de protección de los refugiados políticos de la ONU.

De las relaciones internacionales entre los órganos de represión, salimos para ingresar en los proyectos económicos y de integración regional como política exterior de Estado. Este es el tema del artículo IIRSA, COSIPLAN $\boldsymbol{Y}$

\section{LA ACTUACIÓN BRASILEÑA EN LA CONSOLIDACIÓN DE LA} INTEGRACIÓN DE INFRAESTRUCTURA EN AMÉRICA DEL SUR. El análisis de las iniciativas de integración a partir de proyectos de ingeniería civil e infraestructura sirve como soporte concreto para colocar a prueba la hipótesis del subimperialismo brasileño en América del Sur. Concluye que, si bien hubo beneficios para que las empresas brasileñas se consolidaran internacionalmente a partir de los acuerdos establecidos en el ámbito de la infraestructura y la construcción civil con los países vecinos, no se puede considerar que el propósito de Brasil fuera consolidar un subimperialismo 
económico. Para corroborar esta conclusión, los autores aportan tablas y gráficos estadísticos que dan fe de que los datos de inversión de Brasil son "inexpresivos" en comparación con los de países asiáticos o de la Unión Europea. El artículo está escrito en coautoría de Ana Karolina Morais Silva, Jéssica Maria Grassi y Lucas Kerr Oliveira, especialistas en Relaciones Internacionales en América Latina. Los autores concluyen que la explicación más justa del desempeño brasileño en los países vecinos sería considerar su política externa como un proyecto de liderazgo regional pro integración que se ha desarrollado a lo largo de dos décadas.

El patrón de reproducción capitalista y la lectura crítica sobre la dependencia económica regional son los temas del artículo desarrollado por el doctor en Economía José Alex Rego Soares, bajo el título DEPENDENCIA FINANCIERA LATINOAMERICANA EN EL SIGLO XIX: EL PAPEL DE LA CITY EN EL IMPULSO DE LA INSERCIÓN REGIONAL EN EL SISTEMA FINANCIERO INTERNACIONAL. Se analiza la dependencia regional a partir del siglo XIX, cuando se formaron las nacientes repúblicas, y nuevas economías fueron penetrando progresivamente en el capitalismo que comienza a ser globalizado. Esto ocurrió a través de préstamos e incentivos promovidos por el sistema financiero británico para los regímenes republicanos en América Latina. La lógica de este compromiso con el mercado financiero se habría producido en las circunstancias heredadas del período colonial. Así, el artículo demuestra cómo los lazos económicos con las metrópolis fueron modificados por el régimen del sistema financiero británico y por la relación de esta instancia con los grupos económicos locales. Se concluye que, si por un lado las repúblicas nacientes necesitaban recursos económicos externos, por otro lado el capital financiero contribuyó a generar deudas que, en última instancia, favorecieron el estrangulamiento de las condiciones de desarrollo futuro e de integración regional.

Los siguientes artículos presentados por BJLAS ponen el campo de la geografía en diálogo con estudios sobre ecología política y economía, y con 
debates sobre desarrollo, sociedad y cultura. Esto se debe al hecho de que son artículos que discuten teóricamente y mediante estudios de caso el tema de la agricultura en América Latina, un tema tan antiguo y actual como las tensiones por el territorio y por las autonomías.

En las últimas décadas se han incrementado los estudios sobre agricultura relacionados con crisis socioambientales y culturales. $Y$ al ritmo del tensionamiento que los debates sobre el hábitat y la calidad de vida se imponen sobre la lógica del desarrollo, la gestión pública se ha visto obligada a considerar la agricultura en el contexto urbano como tema central en las políticas de urbanización.

Así, se planteó un desafío, no solo político, sino también teórico sobre las nuevas cuestiones que la urbanización y el medio ambiente han planteado para las ya complejas tareas de la administración pública, obligando a gestores e intelectuales a dialogar con nuevos campos del conocimiento.

Es, pues, del desafío de pensar la agricultura urbana desde nuevos campos de las ciencias sociales y con nuevos instrumentos conceptuales que Henrique Freitas Alves, investigadora en nivel de maestría en Geografía Humana, escribe el artículo ECOLOGÍA POLÍTICA Y AGRICULTURA URBANA EN AMÉRICA LATINA: REFLEXIONES TEÓRICAS PARA UNA APROXIMACIÓN. El argumento que se desarrolla en este trabajo es que la agricultura urbana ha venido exigiendo a los estudios y políticas de urbanización una apertura para incorporar cuestiones fundamentales planteadas por la ecología política urbana. Con una importante sistematización teórica, el artículo ofrece un panorama de los estudios sobre la agricultura urbana como una "forma de reapropiación social de la naturaleza", y es en tal nivel que la ecología política se convierte en una herramienta central para dar respuesta a los dilemas de la distribución desigual del espacio urbano y del derecho a la ciudad. Se trata de nuevos tópicos que nos llevan a pensar filosófica y teóricamente sobre una nueva categoría de justicia: la "justicia espacial". 
En una perspectiva diferente de análisis de la agricultura en América Latina, los siguientes dos estudios nos sitúan ante el avance de la agroindustria y del agronegocio, en áreas campesinas y en nuevas tierras transformadas para la producción intensiva y extensiva para los mercados globalizados. Ambos artículos se complementan así como estudios de caso sobre Colombia, país paradigmático para entender las nuevas ruralidades.

Comenzaremos con los estudios de la investigadora de doctorado Rosa Inés Babilonia Ballesteros y el doctor Julio César Suzuki, ambos del área de Geografía Humana, quienes discuten lo que el pensamiento geográfico llama las nuevas ruralidades, es decir, los efectos de las actividades económicas que transforman los espacios rurales antes integrados por la actividad apoyada en la tradición agrícola de los campesinos. El escenario para este análisis es Colombia, un país que ha experimentado profundos cambios sociales y culturales en las últimas décadas provocados por la transición desde la agricultura campesina a la agricultura comercial y de exportación. Así, el trasfondo histórico es el de la expansión de las políticas de neoliberalización para las zonas rurales, con el consiguiente colapso social y cultural que tal lógica impone a los campesinos. Cabe señalar que el artículo es el resultado de una impresionante investigación con entrevistas e inmersión en el campo para recoger datos cualitativos de gran sensibilidad metodológica que permiten comprender las consecuencias de la nueva ruralidad en los territorios y la vida de las comunidades campesinas. Con este planteamiento, el artículo se titula $\boldsymbol{E} \boldsymbol{L}$

ENFOQUE CUALITATIVO Y SUS APORTES PARA ESTUDIAR EL ESPACIO RURAL: UNA EXPERIENCIA DESDE LA NUEVA RURALIDAD EN COLOMBIA.

El segundo estudio complementa al anterior con un artículo titulado GLOBALIZACIÓN Y RESILIENCIA EN LA SABANA ESTACIONAL DE PUERTO GAITÁN, META, COLOMBIA: REFLEXIONES TEÓRICAS $Y$ METODOLÓGICAS. A partir de inquietudes epistémicas similares -el avance de la agroindustria sobre nuevas fronteras agrícolas- este estudio 
innova al resaltar, con datos históricos, el papel central del gobierno y el de las instituciones internacionales promotoras del desarrollo regional en el suceso de la nueva ruralidad. El segundo aspecto particular de este trabajo es que destaca el avance del capital agroindustrial sobre tierras deshabitadas o consideradas no aptas para la agricultura campesina, justificando la ocupación por parte de la industria agropecuaria basada en la biotecnología. Sin embargo, como demuestra el autor, Carlos Enrique Castro Méndez, doctor en Geografía, si bien estas tierras no son ocupadas tradicionalmente por la agricultura campesina, tienen un lugar en la preservación del ecosistema local y la relación de los seres que allí habitan con su entorno natural. El uso de estas tierras da lugar a formas de segregación espacial y niveles concretos de pérdida de gobernanza territorial, entre otras consecuencias de la desterritorialización producida por los fenómenos económicos globales que provocan daños ambientales imponderables en el futuro.

El último artículo de esta edición de BJLAS también tiene a Colombia como el locus del tema abordado, específicamente la ciudad de Medellín y las políticas de seguridad ciudadana implementadas con resultados concretos en la reducción de la violencia urbana. La tesis que se defiende es que las competencias y la autonomía que se han otorgado a la gestión municipal desde la década de los noventa permitieron las significativas cifras que redujeron, por ejemplo, el 90\% de los homicidios en el municipio. Así, hay un carácter pedagógico fundamental en el análisis de la experiencia de Medellín y que este artículo acertadamente designa en el título: EL ROL DE LA MUNICIPALIDAD EN LA PROMOCIÓN DE LA SEGURIDAD CIUDADANA: POSIBLES LECCIONES DE MEDELLÍN. El aspecto más relevante de este enfoque que ocurre en el campo de las Ciencias Jurídicas y del Derecho es que la autora, investigadora en nivel de maestría Luiza Veronese Lacava, demuestra que la construcción de una política de seguridad exigió como requisito la participación ciudadana bajo la tutela del Acuerdo de Paz, consagrado en 2016, luego de años de tensa 
construcción con participación no solo del gobierno y de las FARC, sino de sectores de la población. Entre otros aspectos, el Acuerdo garantiza mecanismos para proteger la participación popular y las manifestaciones políticas públicas. Por tanto, la seguridad ya no es una cuestión de orden público y de represión sino un producto de los principios de ciudadanía y participación.

Complementando los artículos de BJLAS como siempre, incluimos la reseña de un libro que en este caso fue construido con narraciones ficticias y reales sobre la LITERATURA NAZI EN AMÉRICA LATINA (ROBERTO BOLAÑO). El tema de la reseña encierra impecablemente un número destinado a recordar los siempre actualizados pesares de la violencia estructural que configura las mediaciones políticas entre el Estado y la Sociedad. Esta obra de la literatura latinoamericana fue reseñada por el doctor en Historia Lincoln Secco.

English

\section{A place for memories (Letter to to the readers),}

Finishing 2020, a year of pain and complexity, the Brazilian Journal of Latin American Studies would like to offer to their lectors this new edition of its scientific journal focused on publishing studies about Latin America and the Caribbean in different perspectives that translate the wealth of the region, as well as the complex realities of the countries in our continent.

Thinking about Latin America and the Caribbean requires interpreting the situation of regional dependency, in a world system in which economic and political powers have already defined their positions and movements 
in the international geopolitical board. It also requires a conception of national integration in countries historically marked by colonial injuries, that determine the social hierarchies in which classes relate to criteria of race, gender, nationalist or regionalist feelings, if not religious.

These are the matters addressed by the 38th edition of BJLAS and also the reason for opening the issue with the article THE SARA GÓMEZ'S CINEMA:

\section{A READING ABOUT THE POST-REVOLUTION CONTEXT IN CUBA.}

The inspiring criticism of the film "De Cierta Manera" of the Cuban black filmmaker Sara Gómez is the result of the research work of the PhD in Social History, Cleonice Elias Silva, who has aesthetic and political sensitivity that presents the post-revolutionary scenario of Cuba, and the revolutions that the Revolution failed to accomplish. Based on a film from the 1970s, the author interprets themes that are in the agenda in countries of the rest of the continent: structural racism, gender violence, tensions between the traditional and the modern, as well as other debates about the identities that go beyond the class situation and that underpin the great themes pending on a black, indigenous and diverse Latin America.

The analysis dialogues with black epistemologies and cultural studies, and in this context, it is in line with the two theoretical discussions that we present in this issue of BJLAS. Both approaches, as we will see, have criticisms that the social theory conceptualizes as decolonial. The first is developed in the field of communication and culture. The second focuses on the arenas of history and philosophy.

Let's see the first study, about LATIN AMERICA FOR A DECOLONIAL EPISTEMOLOGY OF COMMUNICATION by Bruno Santos N. Dias, PhD student in Communication Sciences. From the field of Communication and Culture, the author debates classic theories about media production and the cultural industry, whose assumptions instrumentalize the knowledge of the peripheral regions of the system and the cultural creations produced by subalternized peoples. The author takes the 
concept of coloniality of knowledge faced with the Modernity / Coloniality project to the center of the debate on the theories of communication in Latin America and the Caribbean. It also offers as an alternative a dialogical, organic and plural epistemology, anchored in diverse, differentiated and 'enchanted' knowledge. This knowledge is produced in the peripheries of the countries in this region, as intellectual movements of resistance and theoretical, political, cultural and practical disobedience.

\section{In a similar path, Adriana de Carvalho Alves Braga and Christian}

Fernando dos Santos Moura, PhDs in Pedagogy and Arts, expose the Eurocentric project of official history, which makes the victims of colonialism and colonial powers in Latin America invisible and silent. The challenge in this article is to get a dialogue among the decolonial criticisms and the notes of Walter Benjamin in the Conception of History, notes that denounce the primacy of the winners history. The article goes further: it articulates both perspectives with the criticism inaugurated by the revolutionary Marxism of José Carlos Mariátegui, who at the beginning of the 20th century saw the potential protagonist of indigenous people as actors of their political and social transformation. This intellectual exercise results in the eloquent title THE MUTED VOICES: $\boldsymbol{A}$ DEBATE ABOUT COUNTER-HEGEMONIC SOURCES FOR UNDERSTANDING LATIN AMERICA. The intellectual challenge is complete when the authors analyze the chronicles of Guamán Poma de Ayala - the indigenous born in the sixteenth century that describe and draws the colonial violence - as a source of historical data clearly decolonizer and anti-colonial, and as a legitimate and challenging narrative about the official history.

The rescue and challenge to preserve the memory are part of the success of political struggle against historical forms of oppression. The right to memory is a central component of the search for justice for people who have experienced major traumas from episodes of violence, especially by 
the agents of the state, and who with resistance are seeking the transition to a lasting democracy.

It is in this context that the following works should be read, as intellectual and political projects to rescue the memory of one of the most abject episodes of contemporary Latin American history: the promotion of civic-military dictatorships that have been articulately developed in the countries of the region, and that lasted for more than two decades in the mid-twentieth century.

Therefore, it is not surprising that Walter Benjamin also serves as inspiration for the articles that we present as works that rescue the memory because, "the role of the historian is to resume the past of those who were subjugated or defeated, opposing the history presented as progress".

We find this quote in the article PLACES OF MEMORY OF CIVIL-MILITARY DICTATORSHIPS IN LATIN AMERICA, ARCHITECTURE AND SENSE INSCRIPTIONS. The article presents the results of an original research that analyzes the dictatorships legacy from three architectural installations created in Chile, Argentina and Brazil, whose purpose is to leave records and keep alive the memories of regimes that used torture, killings, kidnappings and abductions as a mean of control and state power. The installations are part of the struggle for the consolidation of the fragile democracies of the region and they emerged from the popular demands and the conscious action of the progressive governments of these three countries. The authors who provide us with photographic illustrations and comparative tables of these initiatives are the Master in Science of Information, Caio Vargas Jatene, and Master in Architecture and Urbanism, Luiz de Lucca Neto.

The article by Ana Carolina Contin Kosiak, Master in Law, and Marcos Gonçalves, PhD in History, is also a reminder of the memory facing the Latin American dictatorships. The article is the analysis result of the 
"repression files", records of the Brazilian security agencies on the borders that contain information about the Argentinian people that have fled to Brazil due to the increase of the dictatorship in Argentina at the end of the decade of 1970. The article COOPERATION AND TRANSIT BETWEEN THE ARGENTINIAN AND BRAZILIAN DICTATORSHIP GOVERNMENTS: THE RECORDS OF ARGENTINE REFUGEE'S EXILED TO BRAZIL (1977 - 1979) is noteworthy for its extensive documentary research into records that were prepared by border control agents during a critical period of the Argentine dictatorship. It includes statistical data collected about the composition of the persecuted and refugees, which include women, children and families. The work is also noteworthy because it tracks the less well-known collaboration strategies among Latin American dictatorships to go after their political opponents internationally. In the case of Brazil, the collaboration of the Brazilian military with the Argentine dictatorship took place even after the Brazilian regime had started a political opening and showed willingness to humanitarian policies according to the international organizations for the protection of UN political refugees.

From international relations among the Repression Agencies, we moved to join with economic projects and regional integration as State foreign policy. This is the theme of the article IIRSA, COSIPLAN AND THE BRAZILIAN PERFORMANCE IN THE CONSOLIDATION OF INFRASTRUCTURE INTEGRATION IN SOUTH AMERICA. The analysis of integration initiatives based on civil engineering and infrastructure projects serves as a concrete support for testing the hypothesis of Brazilian sub-imperialism in South America. It concludes despite benefits for Brazilian companies to consolidate themselves internationally based on established agreements in the area of infrastructure and civil construction with the surrounding countries, it cannot be considered that the purpose of Brazil was to consolidate economic sub-imperialism. To support this conclusion, the authors provide tables and statistical graphs that show that Brazil's investment data is "inexpressive" compared with those of Asian 
countries or the European Union. The article is written in co-authorship by Ana Karolina Morais Silva, Jéssica Maria Grassi and Lucas Kerr Oliveira, specialists in International Relations in Latin America. The authors concluded that explaining more justly the Brazilian performance in the neighboring countries would be to consider their foreign policy as a project of regional leadership pro-integration that has developed over two decades.

The capitalist reproduction pattern and the critical lecture on the regional economic dependency are the topics of the article developed by the PhD in Economics José Alex Rego Soares, under the title LATIN AMERICAN FINANCIAL DEPENDENCY IN THE 19TH CENTURY: THE ROLE OF THE CITY IN DRIVING REGIONAL INSERTION IN THE INTERNATIONAL FINANCIAL SYSTEM. The regional dependence is analyzed from the 19th century onwards, when the new Republics were formed, and new economies were gradually entering into globalized capitalism. This happened through loans and incentives promoted by the British financial system for Republican Regimes in Latin America. The logic of this commitment with the financial market would be produced under the circumstances inherited from the colonial period. Thus, the article demonstrated how the economic bonds with the metropolis were modified by the British financial system and the relationship of this instance with the local economic groups. It is concluded that, on one hand, the national republics needed external economic resources, on the other hand, the financial capital contributed to generate debts that, in the last instance, favored the strangulation of the conditions of future development and regional integration.

The following articles presented by BJLAS set the field of geography in dialogue with studies on political ecology and economics, and debates on development, society and culture. This is because they are articles that discuss theoretically and through case studies the topic of agriculture in 
Latin America, which is both old and current, as well as tensions over the territory and autonomies.

In recent decades, studies on agriculture related to the socio-environmental and cultural crisis have been increased. And as the debates about the habitat and the quality of life tensions the logic of development, public management has been forced to consider agriculture in the urban context as a central theme in urbanization policies.

In that way, a challenge was posed about the new questions that urbanization and the environment, not only political but also theoretical, have proposed for the already complex areas of public administration, forcing managers and intellectuals to dialogue with new fields of knowledge.

It is the challenge of thinking urban agriculture from new fields of social sciences and with new conceptual instruments that Henrique Freitas Alves, Master in Human Geography, writes the article POLITICAL ECOLOGY AND URBAN AGRICULTURE IN LATIN AMERICA: THEORETICAL REFLECTIONS FOR AN APPROXIMATION. The argument that is developed in this work is that urban agriculture has been demanding studies and urbanization policies to be open to incorporate fundamental issues raised by urban political ecology. With an important theoretical systematization, the article offers a view of studies on urban agriculture as a form of "social reappropriation of nature", and it is at such level that the political ecology becomes a central tool to answer the dilemmas of the unequal distribution of the urban space and the right to the city. These are new topics that lead us to think philosophically and theoretically about a new category of justice: "spatial justice".

From a different perspective of the analysis of agriculture in Latin America, the following two studies put us before the advance of agroindustry and agribusiness, in rural areas and in new fields transformed for the intensive and extensive production for the globalized markets. Both articles 
complement each other as case studies on Colombia, a paradigmatic country for understanding new ruralities.

We will start with the studies of PhD-level student Rosa Inés Babilonia Ballesteros and Julio César Suzuki, PhD in Human Geography, who discuss what geographic thinking calls the new ruralities, that is, the effects of economic activities that transform rural spaces previously integrated in activities supported by the peasants' agricultural tradition. The scenario for this analysis is Colombia, a country that has experienced deep social and cultural changes in the last decades caused by the transition from peasant agriculture to commercial and export agriculture. Thus, the historical background is the expansion of the neoliberalization policies into rural areas, with the consequent social and cultural collapse that this logic imposed on the peasants. It is worth mentioning that the article is the result of a breathtaking research with interviews and immersion in the field to collect qualitative data of great methodological sensitivity that allows understanding the consequences of the new rurality on the territories and the peasant communities' life. With this focus, the article is entitled THE QUALITATIVE APPROACH AND ITS CONTRIBUTIONS TO STUDY RURAL SPACE: AN EXPERIENCE FROM THE NEW RURALITY IN COLOMBIA.

The second study complements the previous one with an article entitled GLOBALIZATION AND RESILIENCE IN THE SEASONAL SAVANNAH OF PUERTO GAITÁN, META, COLOMBIA: THEORETICAL AND METHODOLOGICAL REFLECTIONS. Based on similar epistemic concerns the advance of agribusiness over new agricultural frontiers - this new study innovates by highlighting, with historical data, the central role of the government and the international institutions that promote regional development in the success of the new rurality. The second particular aspect of this work is that it highlights the advance of agribusiness capital over uninhabited areas or considered unsuitable for peasant agriculture, justifying the occupation by the agricultural industry based on 
biotechnology. However, as the author demonstrates, Carlos Enrique Castro Méndez, PhD in Geography, if these lands are not traditionally occupied by peasant agriculture, they have a place in the preservation of the local ecosystem and the relationship of all inhabitants with their natural surroundings. The use of these lands gives rise to forms of spatial segregation and concrete levels of loss of territorial governance, among other consequences of the deterritorialization produced by global economic phenomena that cause imponderable environmental damage in the future.

The last article of this BJLAS edition also has Colombia as the locus of the approached theme, specifically the city of Medellín and the city security policies implemented with concrete results in the reduction of urban violence. The thesis that is defended is that competences and autonomy that have been given to municipal management since the nineties has allowed significant figures that, for example, reduced $90 \%$ of homicides in the municipality. Therefore, there is a fundamental pedagogical character in the analysis of the experience of Medellín and that this article properly designates in the title: THE ROLE OF MUNICIPALITY IN PROMOTING CITIZEN SECURITY: POSSIBLE LESSONS OF MEDELLIN. The most relevant aspect of this approach in the field of Juridical Sciences, is that the author, master's level student Luiza Veronese Lacava, demonstrates that the construction of a policy of security asked as a requirement for the citizen participation under the tutelage of the Peace Agreement, which was finally consecrated in 2016, after years of tense construction. This was reached with participation of the government, the FARC and sectors of the population. Among other aspects, the agreement guarantees mechanisms to protect popular participation and public political manifestations. Therefore, security is no longer a matter of public order repression but a product of the principles of citizenship and participation.

Complementing the articles of BJLAS as always, we include the review of a book that in this case was built with fictional and real narrations about the 
NAZI LITERATURE IN LATIN AMERICA (ROBERTO BOLAÑO). The theme of the review impeccably closes a number designed to remember the always up to date regrets of structural violence that shape political mediations between the State and Society. This work of Latin American literature was reviewed by the PhD in History Lincoln Secco.

DOI:10.11606/issn.1676-6288.prolam.2020.180488

Recebido em: 30/12/2020 Aprovado em: 30/12/2020 Publicado em: $30 / 72 / 2020$ 